\title{
Frequency sensitivity of nanosecond pulse EMF on regrowth and hsp70 levels in transected planaria
}

\author{
Ash Madkan ${ }^{1 *}$, Avary Lin-Ye1, Spiro P. Pantazatos ${ }^{2}$, Matthew S. Geddis ${ }^{3}$, Martin Blank², Reba Goodman ${ }^{1}$ \\ ${ }^{1}$ Department of Pathology and Cell Biology, Columbia University College of Physicians and Surgeons, New York, USA; \\ ${ }^{2}$ Department of Physiology and Molecular Biophysics, Columbia University College of Physicians and Surgeons, New York, USA; \\ ${ }^{3}$ Department of Science, Borough of Manhattan Community College-CUNY, New York, USA. \\ Email: rmg5@columbia.edu
}

Received 30 June 2009; revised 10 July 2009; accepted 15 July 2009

\section{ABSTRACT}

Purpose: To study the effect of time varying/ pulsing electromagnetic fields (PEMF) on biological systems by measuring regrowth and the induction of elevated levels of the stress protein hsp70 in the regenerative model Planaria Dugesia dorotocethala. Objective: The outcomes of studies using electromagnetic fields (EMF) are dependent on pulse design, field strength (mG), frequency $(\mathrm{Hz})$, duration and magnetic field/rise time $(\mathrm{dB} / \mathrm{dt})$. Standardization of effective pulse design is necessary to avoid continuing confusion in the investigation of pulsing electromagnetic field (PEMF) technology. Information from studies on hsp70 protein induction and regrowth in transected Planaria provides information on EMF efficacy for potential clinical application in the treatment of ischemia reperfusion injury and the eventual inclusion of EMF prophylaxis prior to surgery. Materials and methods: Planaria were transected equidistant between the tip of the head and the tip of the tail. Individual head and tail portions from the same worm were placed in pond water and exposed to 8,16 or 72 Hertz PEMF for one hour daily post transection under carefully controlled exposure conditions. Regrowth of heads and tails was measured in PEMF-exposed and sham control. Protein lysates from PEMF-exposed and sham control transected heads and tails were analyzed for hsp70 levels by Western blot analyses. Conclusion: The degree of regrowth and hsp70 levels in transected heads and tails exposed to nanosecond PEMF exposures at 8,16 or $72 \mathrm{~Hz}$ was frequency dependent. There are currently several views on the interaction mechanism involved in regrowth. Here we discuss two: in

Abbreviations: Extremely low frequency (ELF), electromagnetic fields (EMF); nanosecond pulsing electromagnetic field (PEMF), heat shock protein (hsp), heat shock gene (HSP), frequency (Hz), Gauss (G), magnetic field/rise time (dB/dt); mitogen-activated protein kinase (MAPK). one $[7,8]$ we propose a direct effect on the DNA of the PEMF consensus sequence, nCTCTn, referred to as electromagnetic field response elements (EMRE) in the promoter region of the stress response gene HSP70. In the second mechanism [28] it is proposed that EMF induce vibrations of proteins through a series of quantized low frequency phonon signals.

Keywords: Planaria; Nanosecond EM Pulse; hsp70 Protein; Regrowth

\section{INTRODUCTION}

Electromagnetic fields (EMF) of less than 1 Gauss strength have been shown to induce a variety of specific effects in cells and tissues over a wide frequency range of the EM spectrum $[7,8]$. Nanosecond pulsing electromagnetic field (PEMF) technology has assumed increasingly greater importance in clinical treatments $[15,29,70]$. As a measure of efficacy, we used the regenerative model Planaria, to test three nanosecond PEMF devices on regrowth of transected heads and tails and the induction of the stress response protein hsp70 [42,43,44,45].

Previously we showed that ELF-EMF induce elevated hsp70 protein levels through the responsiveness of a specific consensus DNA sequence EMRE (electromagnetic field response element, nCTCTn) on the heat shock 70 (HSP70) promoter $[41,42,43,44,45]$. This DNA domain is upstream from the heat shock domain. Furthermore, ELF-EMF induction of the hsp70 protein protects cells and limits the effects of subsequent stress, including sudden changes in temperature [12,30].

The degree of electromagnetic field-effects on biological systems is known to be dependent on a number of criteria in the waveform pattern of the exposure system used; these include frequency, duration, wave shape, and relative orientation of the fields $[6,29,32,33,39,40]$. In some cases pulsed fields have demonstrated increased efficacy over static designs $[19,21]$ in both medical and experimental settings. 
To examine the effects of PEMF on regrowth following transcetion and the induction of hsp70 protein in transected heads and tails, Planaria, a Platyhelminth, were used as a model organism because of its recognized ability to regenerate [55]. Previous studies showed that EM sinusoidal fields enhance the first three to six days of regeneration in Planaria and during this time specific proteins in the MAPKinase cascade are activated [26]. Planarians regenerate through a well defined stem cell system by restimulating mechanisms that guide the patterning of body structures during embryonic development $[1,2,3,13,14,56,57,58,59]$. Planaria are typically able to regenerate into a full worm from any body part within 14-16 days [55,32,33] thus providing a sensitive model for examining how electromagnetic fields interact with specific stages of regeneration.

The potential application of the upregulation of the HSP70 gene by both ELF-EMF and nanosecond PEMF in clinical practice would include trauma, surgery, peripheral nerve damage, orthopedic fracture, and vascular graft support, among others. Regardless of pulse design, EMF technology has been shown to be effective in bone healing [5], wound repair [11] and neural regeneration $[31,36,48,49,51,63,64,65,66]$. In terms of clinical application, EMF-induction of elevated levels of hsp70 protein also confers protection against hypoxia [61] and aid myocardial function and survival $[20,22]$. Given these results, we are particularly interested in the translational significance of effect vs. efficacy which is not usually reported by designers or investigators of EMF devices. More precise description of EM pulse and sine wave parameters, including the specific EM output sector, will provide consistency and "scientific basis" in reporting findings.

\section{MATERIALS AND METHODS}
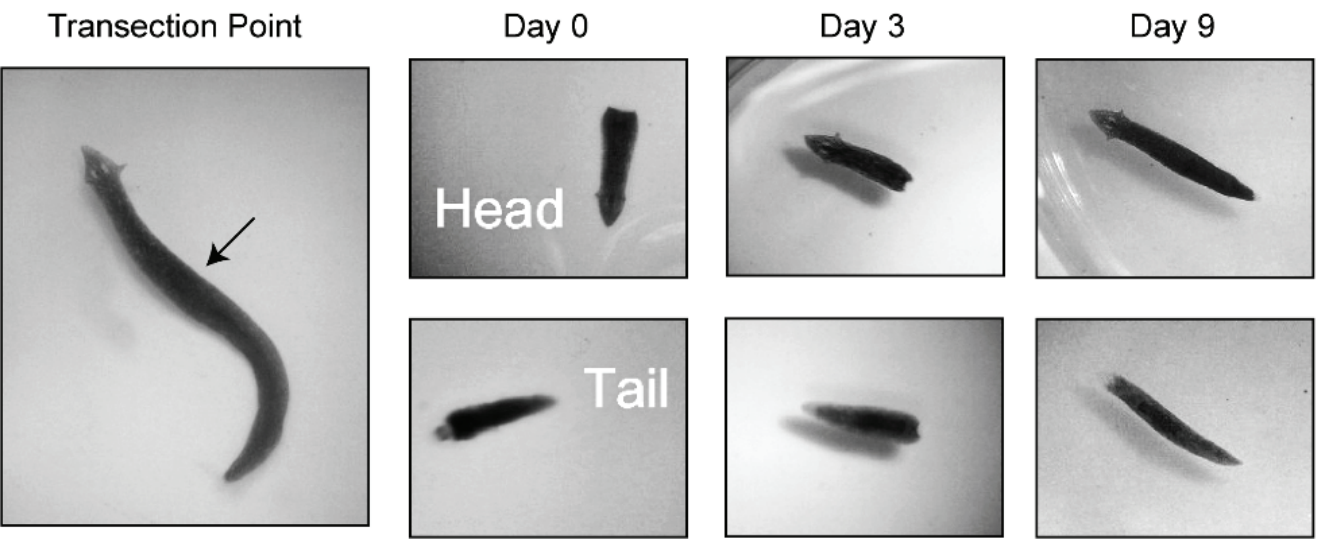

Planaria. Dugesia dorotocethala (Carolina Biological Supply Company; cat. \# 132950) were shipped overnight and allowed to 'recover' for at least 24 hours in fresh oxygenated pond water (Carolina Biological Supply Co, $\mathrm{NC}$, USA). Planaria were maintained in near darkness at 22 to $24^{\circ} \mathrm{C}$ (Precision Scientific incubator; Fisher Scientific, NJ, USA) throughout the experiments.

\subsection{Experimental Protocol}

Planaria Dugesia dorotocethala were transected equidistant between the tip of the head and the tip of the tail (Figure 1). The following is the experimental protocol used in these experiments:

1) Following transection ( 0 time) head and tail portions were exposed to 8,16 or $72 \mathrm{~Hz}$ nanopulse for one hour twice a day. Measurements of regrowth were performed from 0 time to at least three day intervals $(\mathrm{n}=>10$ experiments).

2) Following transection, heads and tails were exposed to 8,16 or $72 \mathrm{~Hz}$ up to 180 minutes. Samples were removed at 20 minute intervals for protein extraction and hsp70 analyses $(\mathrm{n}=>10)$.

3) Following transection, heads and tails were exposed to 8,16 or $72 \mathrm{~Hz}$ fields for one hour twice per day over a 12 day time period. Ten head and tail samples were removed daily and prepared for hsp70 analyses. Experiments were repeated at least three times.

Exposure protocol. In the experiments described here, Planaria Dugesia dorotocethala were transected equidistant between the tip of the tail and the head (Figure 2). Each head and tail portion was photographed using a Nikon digital camera mounted on a Wilde dissecting microscope. Images were stored in a database for subsequent measurements using ImageJ (see section on Quantitation). Head and tail portions were placed in

Figure 1. Transection of Planaria. The image in the left panel shows an intact sham exposed planaria with head facing up. The arrow indicates the transection point. The adjacent panels show representative images of heads and tails from transected Planaria at days 0,3 , and 9. The images have been enlarged 16X to show detail. 


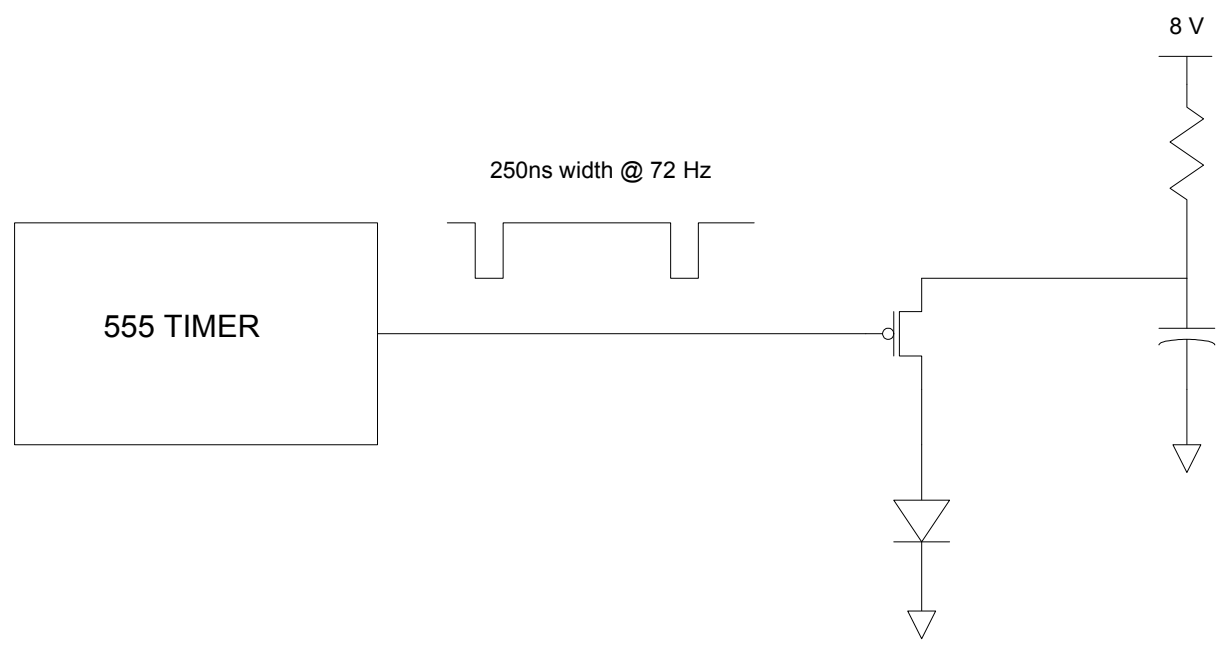

Figure 2. EM Probe Device. This device is designed to generate fast magnetic field pulses of low duty cycle in the near field region. The pulse length is about $250 \mathrm{~ns}$ driven by a storage capacitor and a MOFSET switch that produces a $10 \mathrm{~A}$ current in a field generating circuit element. The circuit element is just the single loop of the capacitor and switch circuit and does not contain any additional coil or turns of a conductor to enhance the magnetic field strength. The compact single loop design minimizes inductance and increases the speed of the circuit allowing a fast rise pulse producing a maximum magnetic field of the order of one Gauss at about $1 \mathrm{~cm}$ from the device. The field is concentrated in a region of a few $\mathrm{cm}^{3}$ and falls off rapidly outside that region. The fast rise and fall of the current and magnetic field pulse implies a broad spectrum of Fourier components contain within the repeated pulse of the waveform. For the pulse rise time of about $10 \mathrm{~ns}$ and a pulse frequency of $72 \mathrm{~Hz}$, those components extend from the fundamental frequency at $72 \mathrm{~Hz}$ at the low end to at least $20 \mathrm{MHz}$ at the high frequency end. The particular distribution of frequencies is determined by the usual methods of Fourier analysis from the exact pulse shape. This wide range of frequency offer resonant interactions with the biological mechanisms of the organism being treated over a very wide range and could include molecular, cellular and multicellular level interactions.

separate Petri dishes (Falcon $35100760 \times 15 \mathrm{~mm}$; Fisher Scientific, NJ, USA) containing pond water approximately $0.6 \mathrm{~cm}$ deep. Dishes were numbered so that heads and tails of the same worm could be identified for measurements. Dishes were placed on a firm base and the PEMF devices were attached to the dishes so that the entire area of the dish was exposed to a uniform field for one hour a day. All exposures took place within an incubator with the temperature maintained at $22-24^{\circ} \mathrm{C}$ and monitored with a thermocouple probe (sensitivity +/$0.01^{\circ} \mathrm{C}$; Physitemp, model BAT12, Hackensack, NJ, USA). Growth was assessed at three-day intervals from day 0 (immediately following transection and the onset of PEMF exposure) to day 12 post-transection. In a series of separate experiments, pigmented eye spot development was monitored at 12 hour intervals in transected tails exposed to PEMF and sham control immediately following transection.

The nanosecond PEMF device (EM-PROBE Technologies) in Figure 1 generates a near field fast magnetic pulse of 250 nanosecond (ns) duration, which is driven by a circuit containing a storage capacitor and a switch that produces a $10 \mathrm{~A}$ current in a field-generating circuit element. The single loop circuit does not contain any additional coils of a conductor to enhance the magnetic field strength, and allows a rapid $\mathrm{dB} / \mathrm{dt}$ pulse design producing a magnetic field of 1 Gauss approximately $1 \mathrm{~cm}$ from the device. The pulse begins with a peak strength of
1.4-1.7 Gauss that deteriorates to zero in about 200ns. At $72 \mathrm{~Hz}$ this means that there is an active field for $0.00072 \%$ of the time. At $16 \mathrm{~Hz}$ there is an active field for $0.00016 \%$ of the time. At $7.8 \mathrm{~Hz}$ there is an active field for $0.000078 \%$ of the time. The particular distribution of frequencies is determined by Fourier analysis from the exact pulse shape. This wide range of frequencies offers resonant interactions with the biological mechanisms of the organism being treated. The entire output circuit is optimized for rapid response by minimizing inductance. The wide range of frequencies offers resonant interactions with the biological mechanisms of the organism being treated over a wide range and might include molecular, cellular and multicellular interactions.

Shielding: Both active (experimental) and sham- exposed (control) samples were enclosed in $30 \mathrm{~cm}$ high, $15 \mathrm{~cm}$ diameter cylindrical $\mathrm{Mu}$ metal containers $\left(0.040^{\prime \prime}\right.$ thickness) (Amuneal Corp. Philadelphia, PA). Detailed measurements of background magnetic fields in the incubator, harmonic distortion, DC magnetic fields and mean static magnetic fields in the incubators were previously determined [35].

Protein lysates. In a separate series of experiments, protein was extracted at defined time periods from the transected heads and tails, both experimental and sham, to determine effect of PEMF on hsp70 levels. Lysates were prepared from the heads and the tails of exposed and sham exposed Planaria for analyses of hsp70 [41, 42, 
45]. Protein concentrations were determined by the Bradford assay (Bio-Rad Redmond, WA, USA).

Western blot. Lysed samples containing $30 \mu \mathrm{g}$ of protein were subjected to sodium dodecyl sulfate gel electrophoresis (Fisher Scientific, NJ, USA) on $10 \%$ polyacrylamide gels using appropriate molecular weight markers (Santa Cruz Biotechnology Inc, Santa Cruz, CA, USA) and the polypeptides were transferred to polyvinylidene fluoride membrane for immunoblotting. Blots were probed with anti-hsp70 antibody $(1: 10,000)$. The blots were then stripped using SuperSignal West Pico Stable Peroxide Solution (Pierce, prod \# 1859674, Fisher Scientific, NJ, USA) and SuperSignal West Pico Luminol Enhancer Solution (Pierce prod \# 1859675, from Fisher Scientific, NJ, USA) and reprobed with anti-actin (1:1000) to confirm equivalent loading. Visualization was by the enhanced chemiluminescent detection system (Fisher Scientific, NJ, USA) as previously described [43].

Antibodies. The antibody to hsp70 was kindly provided by Dr. Richard Morimoto, Northwestern University.

Statistical analysis: For determination of regrowth, multiple high-resolution digital pictures of Planaria heads and tails were taken at three day intervals and the rate of regrowth quantified using ImageJ v1.38 (http:// rsb.info.nih.gov/ii). Length measurements were calibrated in millimeters and transposed to an Excel spreadsheet for statistical analysis. Lengths were normalized to the 0-time. Length differences over each 3-day interval were then subjected to 2-sample t-tests to assess significant differences in mean growth between the control and exposed conditions for both heads and tails. An additional analysis was conducted in SAS v9.1 (www. sas.com) to assess the significance of an interaction effect, i.e., whether the exposure had greater effect on either heads or tails. For this a 'mixed effects' analysis was performed which modeled the average length at 3-day intervals as a linear sum of the fixed effects of exposure (experimental vs. control), worm portion (heads vs. tails) and their interaction.

For quantification of the hsp70 and beta-actin levels the films from Western blot were scanned and saved as digital images (Figure 3). The densities of the bands were quantified using the histogram function in ImageJ and values transposed to Excel for statistical analysis (2 sample t-tests). A minimum of three replications of each assay were conducted.

\subsection{Western Blots Quantitation of Hsp70 and $\beta$-actin Bands}

Images from films were scanned into a computer and analyzed with Image J v1.37 (NIH). The analyze function for gels was used to plot the spatial signal density for each lane of hsp70 (see Figure 2). The same was done for the $\beta$-actin controls (not shown). Figure 2 plots the mean hsp70/-actin ratios for PEMF and Control conditions. These values were imported into a Microsoft Excel spreadsheet where the signal value of hsp70 was divided by the value of the-actin signal in the same lane in order to normalize against variable loading volumes. -actin is a housekeeping gene and unaffected by EMF or PEMF. Statistical analyses were also conducted in Excel using the Data Analysis toolbox.

\section{RESULTS}

The extraordinary ability of Planaria to regenerate after injury is attributed to the presence of totipotent neoblasts capable of differentiating into all of the tissue types [1, 4]. Perhaps most impressive is their ability to regenerate the nervous system. When transected the tail region forms a new head complete with bilateral optic nerves and eyes, a two-lobed brain, and a pair of ventral nerve cords (VNC) $[2,13,14]$.

\subsection{PEMF Accelerates Rate of Regrowth of Transected Planaria}

To assess the efficacy of the nanosecond PEMFs on Planaria regeneration, the length of ELF-EMF-exposed and sham control heads and tails were measured at threeday intervals starting immediately following transection (Figure $4 \mathrm{~A}, \mathrm{~B}, \mathrm{C}$ ). The heads and tails of all transected Planaria (experimental and control) regrew to normal viable worms. The initial experiments used PEMF at $72 \mathrm{~Hz}$.to measure regrowth of transected heads and tails. As previously demonstrated using ELF-EMF [26], accelerated tail regrowth was significant at days 3 and 6 post transaction $(p=0.05)$. In contrast to the effect of exposure to $72 \mathrm{~Hz}$ PEMF, $8 \mathrm{~Hz}$ PEMF exposure on the tail portion showed the greatest response to any of the

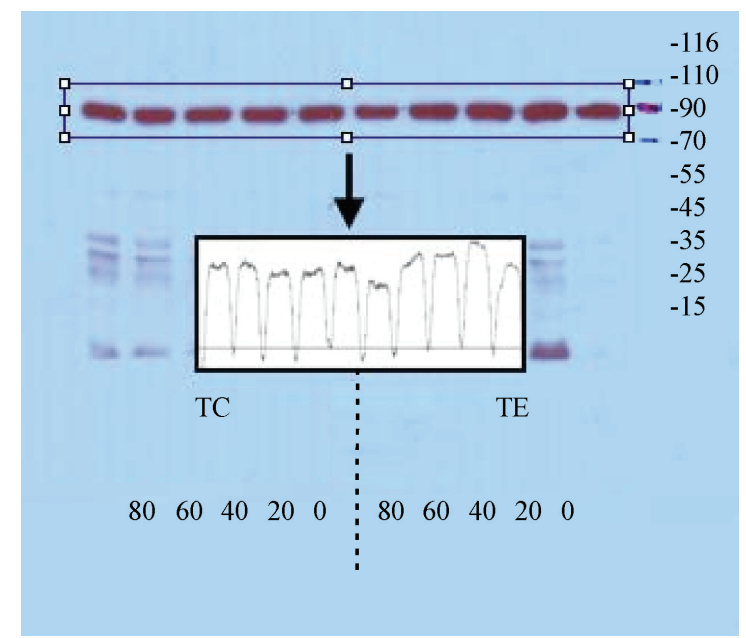

Figure 3. Images from films were scanned into computer and analyzed with Image J v1.37 (NIH). The 'analyze function for gels' was used to plot the spatial signal density for each lane of hsp70. The same was done for the $\beta$-actin controls (not shown). These values were imported into a Microsoft Excel spreadsheet where the signal value of hsp70 was divided by the value of the $\beta$-actin signal in the same lane in order to normalize against variable loading volumes. $\beta$-actin is a so-called 'housekeeping gene' and unaffected by EMF or EMP. Statistical analyses were also conducted in Excel using the Data Analysis toolbox. 
frequencies to which the transected heads and tails were exposed. The sham control samples in general showed very little change in length over this time period (Figure
3B). A reduced response to the $16 \mathrm{~Hz}$ PEMF (Figure 3C) was measured in both transected heads and tails when compared with 72 and $8 \mathrm{~Hz}$ (Figure 3A, B).

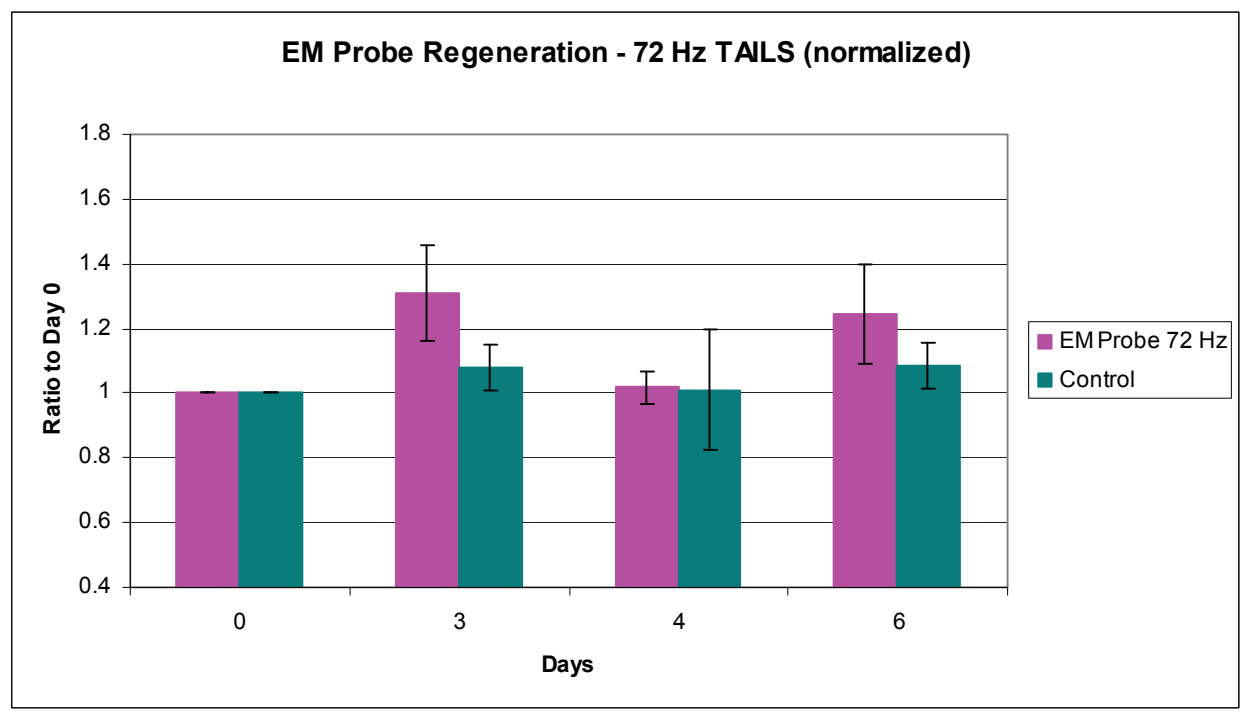

Figure 4A. Regeneration of heads and tails following transection of Planaria. Histograms showing the mean length of control and PEMF-exposed heads and tails immediately post-transection $(0$ time), and 3 to 9 days post-transection. (A) Mean length \pm 2 standard error of the mean (SEM) of exposed heads (HE, $n=48)$ and sham exposed heads ( $\mathrm{HC}, \mathrm{n}=27)$ at each time. (B) Mean length $\pm 2 \mathrm{SEM}$ of exposed tails (TE, $n=20)$ and sham exposed tails $(\mathrm{TC}, \mathrm{n}=12)$ at each time. The asterisks indicate a significant statistical difference in the rate of regeneration between exposed and sham exposed heads and tails assessed by 2 -sample t-tests of mean difference in length.

A. Exposure to $72 \mathrm{~Hz}$ accelerated tail regrowth was significant at days 3 and 6 . post-transection [data normalized against day zero $(n=3)]$. The effect of the $72 \mathrm{~Hz}$ EM probe is 'statistically significant $(\mathrm{p}=0.05)$ at three and six days on the tail portion. The heads and tails of all transected Planaria (experimental and control) regrew to normal viable worms by 20 days. There was little or no measureable effect on regrowth in head portion.

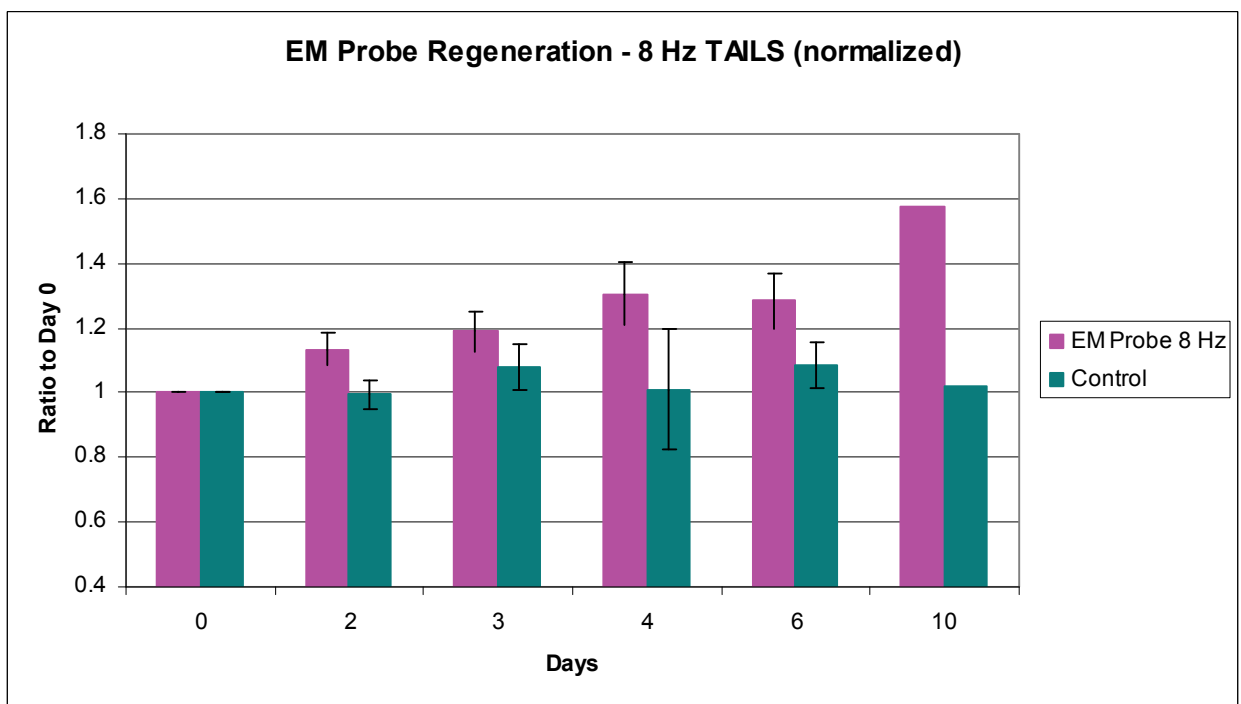

Figure 4B. In contrast to the effect of exposure to $72 \mathrm{~Hz}$ PEMF, transected tails exposed to the $8 \mathrm{~Hz}$ EM probe show a steady increase in length. The effect of $8 \mathrm{~Hz}$ PEMF on the tail portion showed the greatest response to any of the frequencies to which the transected heads and tails were exposed. The sham control samples and the head exposed samples showed very little change in length over this time period. 


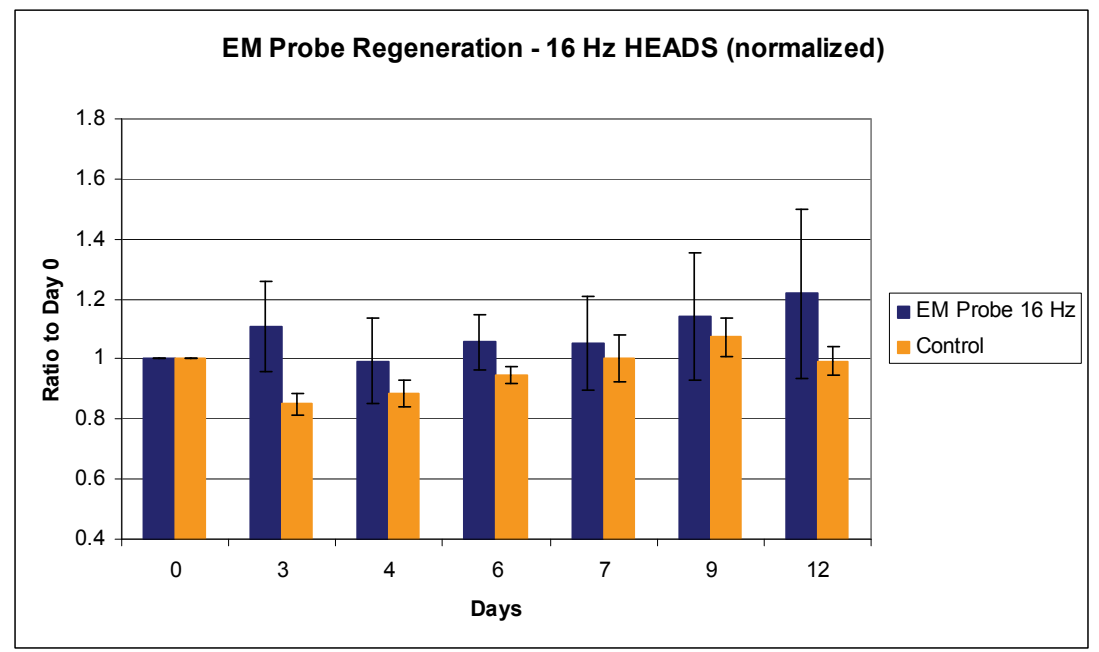

Figure 4C. A reduced response to the $16 \mathrm{~Hz}$ PEMF was measured in both transected heads and tails when compared with 72 and $8 \mathrm{~Hz}$.

Of particular interest was the general trend of the responsiveness of the tail to the three frequencies tested as compared to the head portions (Figure 4 A, B, C). The third day after transection has been shown to be the time of maximal ELF-EMF-stimulated growth of the tail [26]. During normal regeneration this is the developmental stage when the neural networks are actively forming [13, 14] and the ventral nerve cords (VNC) are extending from the head into the newly formed tail.

\subsection{PEMF Activation of Hsp70 Associated with Injury and Repair}

A significant elevation in hsp70 levels was evident in tail portions that were exposed to the nanosecond PEMF using all three frequencies at 0,20, 40, 60 and 80 minutes (Figure 5). We also looked at potential differences in induction of hsp70 protein, comparing all separate PEMF exposures to the sham control. Tails that were exposed to $16 \mathrm{~Hz}$ EM-Probe fields showed the highest hsp70 production ( $\mathrm{p}=.000007 .91)$.

In terms regrowth, the $8 \mathrm{~Hz}$ tails shows the quickest response to PEMF (Figure 4B). The $72 \mathrm{~Hz}$ also shows a noted difference between exposed and unexposed worms (Figure 4A). The $16 \mathrm{~Hz}$ was too inconsistent in order to draw any conclusions (data not shown).

We believe the accelerated regrowth above is consistent with the role of hsp70 as a chaperone that monitors the folding of proteins during repair. PEMF induction of the HSP70 gene may result from events triggered by the activation of ERK that utilize a unique consensus sequence (electromagnetic field response element; EMRE) upstream from the heat shock consensus sequence $[8$, $9,10]$.

The increase paralleled the accelerated regeneration noted above and is consistent with the role of hsp70 as a chaperone that monitors the folding of proteins during repair. Induction of the HSP70 gene may result from events triggered by the activation of ERK that utilize a unique consensus sequence (electromagnetic field response element; EMRE) upstream from the heat shock consensus sequence [42,47]. Stress response protein hsp70 levels are elevated after exposure to PEMF [43, 44,45]. We next examined the effect of nanosecond PEMF at 8, 16 and $72 \mathrm{~Hz}$ exposures on levels of this protein. Earlier studied had shown that hsp70 levels (Figure 5A,B) depicts mean hsp70/-actin ratios and 95\% CI for both $16 \mathrm{~Hz}$ and $72 \mathrm{~Hz}$ exposure conditions on heads and tails respectively vs. their control counterparts. With both frequencies we observed a spike in hsp70 levels at 40 minutes which concurs with our previous data $[41,45]$ and as previously noted the hsp70 levels return to normal levels by 120 minutes. [Mean hsp70/-actin ratios for $16 \mathrm{~Hz}$ EMP Heads $(\mathrm{n}=14), 16 \mathrm{~Hz}$ EMP Tails $(\mathrm{n}=14), 72 \mathrm{~Hz}$ EMP Tails $(\mathrm{n}=17)$ and $72 \mathrm{~Hz}$ EMP Heads $(\mathrm{n}=13)$.] As expected, measurements of hsp70 during regrowth showed little to no effect of exposures.

\section{DISCUSSION}

In the experiments reported here we examined nanosecond PEMF, rapid $\mathrm{dB} / \mathrm{dt}$ using 8,16 and $72 \mathrm{~Hz}$ EM probe devices on induction of hsp70 levels and Planarian head and tail regrowth post-transection. Our data show that the induction of elevated hsp70 levels and regrowth following transection are frequency specific; tail portions that regenerate nervous system, brain and eyes, are more responsive to the EM probe device than head portions. The effectiveness of time varying electromagnetic fields on biological systems has been shown to be dependent on pulse design; frequency, duration, magnetic field/rise time $(\mathrm{dB} / \mathrm{dt})[29,30]$. Measurements of EMF induced 
Planaria EM Pulse Solo - $16 \mathrm{~Hz}$ and Control

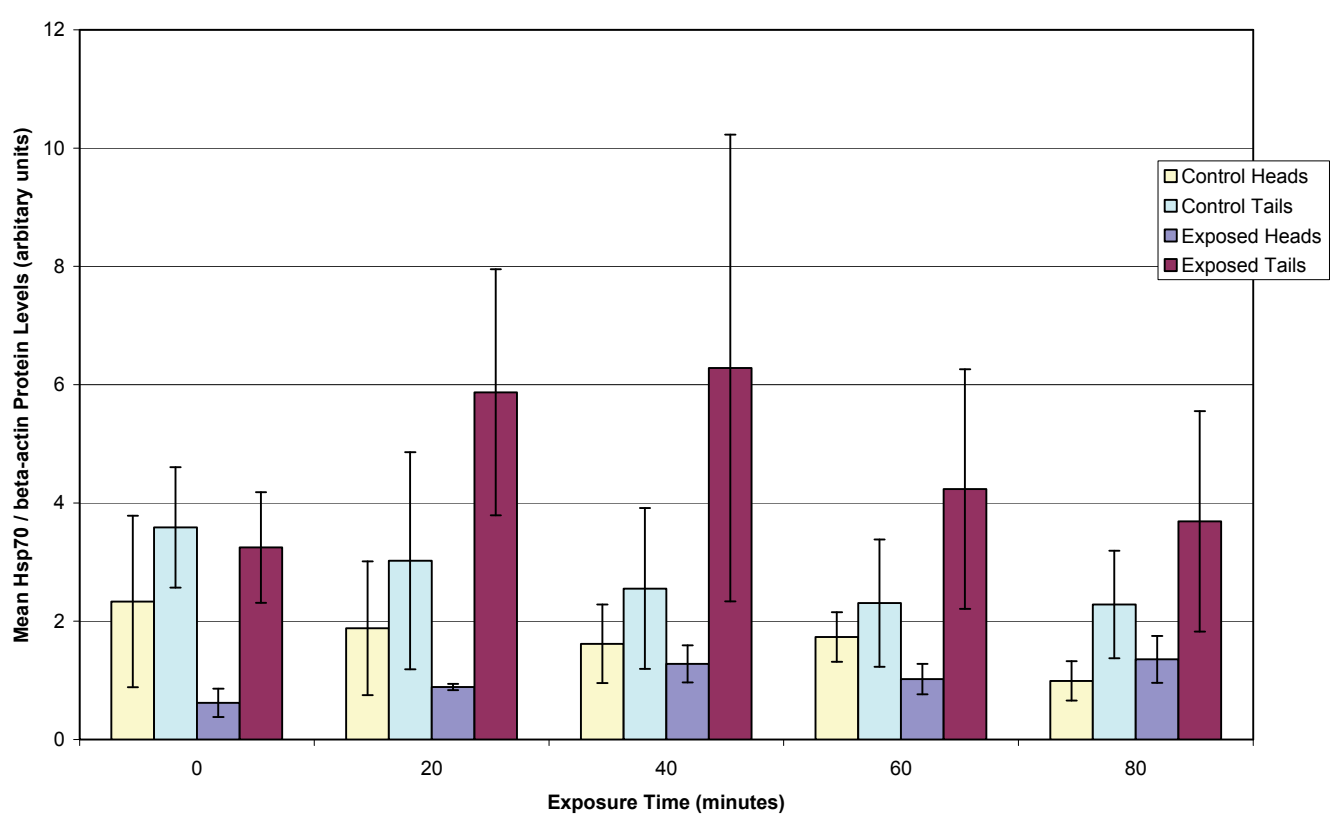

Figure 5A. Histogram showing a quantitative analysis of hsp70 protein levels from regenerating heads, tails, and sham controls. The relative protein levels are expressed in arbitrary units based on the intensity of the hsp70 protein band from the Western blots. The PEMF exposed tails show an obvious spike between 20 and 40 minutes. Mean hsp70/ $\beta$-actin ratios for $16 \mathrm{~Hz}$ PEMF heads $(\mathrm{n}=14), 16 \mathrm{~Hz}$ PEMF tails ( $\mathrm{n}=14), 72$ $\mathrm{Hz}$ PEMF tails $(\mathrm{n}=17)$ and $72 \mathrm{~Hz}$ PEMF heads $(\mathrm{n}=13)$. The control groups for each sample had a sample size equal to that of its corresponding PEMF group. Error bars represent $95 \%$ confidence intervals $( \pm$ $2 *$ Standard Error).]

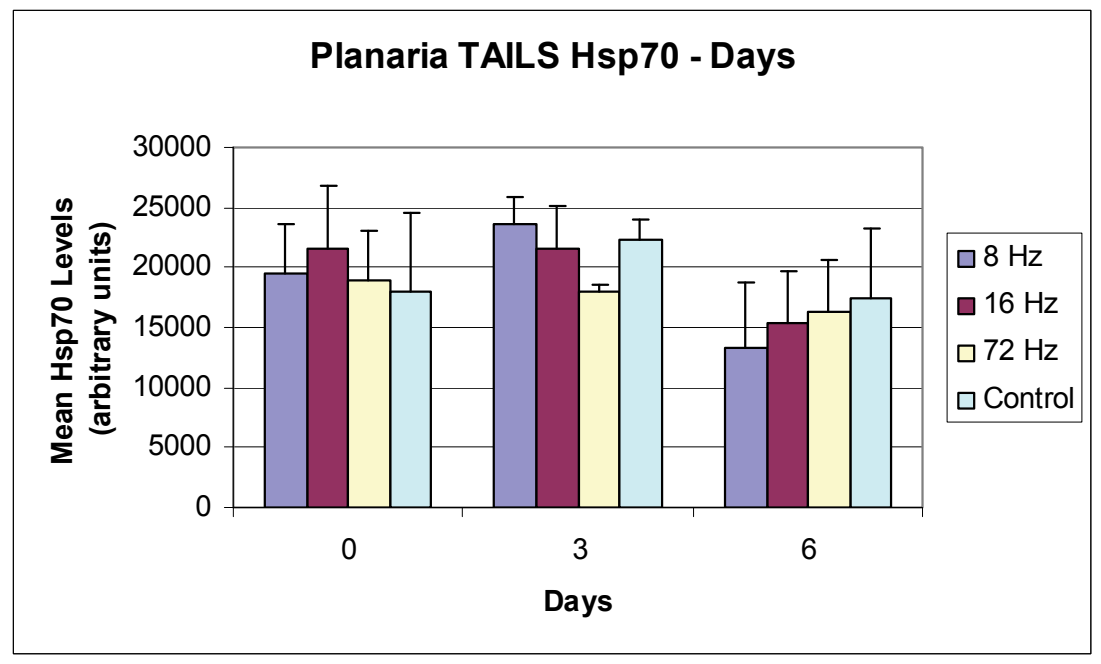

Figure 5B. Histogram showing a quantitative analysis of hsp70 protein levels from regenerating heads, tails, and sham controls.[Mean hsp70/ $\beta$-actin ratios for $16 \mathrm{~Hz}$ EMP heads $(\mathrm{n}=14), 16 \mathrm{~Hz}$ PEMF tails ( $\mathrm{n}=14), 72$ $\mathrm{Hz}$ PEMF tails $(\mathrm{n}=17)$ and $72 \mathrm{~Hz}$ PEMF heads $(\mathrm{n}=13)]$. The control groups for each sample had a sample size equal to that of its corresponding EMP group. Error bars represent $95 \%$ confidence intervals $( \pm$ $2 *$ Standard Error).]

hsp70 levels and induction of post-transected re-growth were used as markers for repair efficacy in this study.
Standardization of effective pulse design is necessary to avoid continuing confusion in the investigation of puls- 
ing electromagnetic field (PEMF) technology. The data from hsp70 protein induction and regrowth in Planaria, a regeneration model, as a measure of EMF efficacy, suggest excellent opportunity for clinical use in treatment of ischemia reperfusion injury and the inclusion of EMF prophylaxis prior to surgery. Our focus in these experiments was on: (a) regrowth of head and tail portions following transection, (b) immediacy of hsp70 induction during regeneration of heads and tails, (c) levels of hsp70 elevation during regeneration of heads and tails and (d) duration of hsp70 elevation. We have shown that the tail portion of the transected Planaria is more sensitive to different frequencies both in the induction of hsp70 levels and in regrowth. Furthermore, these changes in tail metabolism occur at different times depending upon the frequency used in the exposure.

\subsection{Interaction Mechanisms}

How weak electromagnetic (EM) fields interact with DNA to stimulate protein synthesis remains unknown; EMF interaction with cells and tissues has been examined in a variety of in vivo and in vitro systems including enzyme reactions [10], Drosophila [25,27,72], yeast [71] cultured cells $[23,24,53]$ and on regeneration in Planaria [26]. Currently no definitive mechanism exists to explain how EMF interact with DNA and proteins in cells and tissues, although several theories have been proposed including cyclotron resonance [40], and a modification of this idea [6,37].

There is some evidence that EM fields can affect DNA, both directly and indirectly [9]. We have shown a unique DNA sequence in a specific domain on the HSP70 promoter that is sensitive to EM field exposures and this observation has contributed to the understanding, in part, of how ELF-EMF affects biological systems. There are specific regions on the promoters of some genes, HSP70 and c-myc for example, that contain this consensus sequence and when this sequence is transfected into promoter of reporter genes, these genes become sensitive to EMF, whereas before they were not. $[41,42,43,44,45]$. EM fields initiate up regulation of the HSP70 gene, increase mRNA transcripts and hsp70 protein levels. The responsiveness is dependent on the number of copies of the nCTCTn consensus sequence. [42]. Electromagnetically, responsive elements act as 'sensors'. This is an HSF-1 dependent process as shown by electrophoretic mobility shift assays (EMSA) of protein extracts from HL60 cells exposed to EMF. HSF1DNA binding activity was demonstrated by a supershifted band. The magnetic field-inducible heat shock element-binding activity is HSE sequence-specific and contains HSF1 [45].

Because of the demonstrated effect of EMF on electron transfer reactions, it has been proposed that displacement of electrons in the H-bonds that hold DNA together can lead to DNA chain separation, thus initiating transcription $[7,8]$. The resultant charging due to electron displacement on the dynamics of DNA chain separation suggests that electron transfer could favor separation of base pairs e.g. nCTCTn, with the EM field sensitive DNA sequence acting as the first order iterative mechanism. In the case of Planaria the DNA in the totipotent stem cells created by the injury may be responding directly to the EM fields. It has been shown that DNA-mediated charge transport and the oxidative damage that results are extremely sensitive to variations in the sequence and conformational-adaptive response leading to stacking of the intervening bases $[18,62]$. Protein-binding to DNA, for example, through one or more of the MAPKinase cascades, modulates long-range charge transport negatively and positively depending upon the specific protein DNA interactions in play $[52,54,68,69]$.

A possible mechanism for EMF initiation of protein synthesis is by acting on the de-localized $\pi$ electrons in the base pairs that hold the DNA strands together. This is one way in which the effect of EMF in ELF range can be due to the resulting current. The charging of molecular complexes has been shown to lead to their disaggregation [10], so local charging would be expected to cause the DNA strands to disaggregate. A possible sequence of steps may start with EMF displacing electrons and charging DNA segments, followed by disaggregation of DNA strands as a result of the charging.

The properties of the CTCT bases suggest that they may be involved in the first step in a molecular mechanism for EMF activation of protein synthesis. They have low electron affinities, so electrons would be more easily displaced by the EMF. Also, the CTCT are pyrimidines, and when the H-bonds split between CTCT and the GAGA (purines) bases on the complementary chain, the smaller area that results would require less energy, and make the disaggregation more favorable [7].

Two recent studies of molecular properties of DNA add support to this proposed mechanism of DNA disaggregation. One paper compared the lifetimes of excitation induced by ultraviolet (UV) light in different DNA structures [60]. Apparently, the induced fluorescence had significantly longer lifetimes in DNA chains composed of $A$ and $\mathrm{G}$ bases than in chains with $\mathrm{C}$ and $\mathrm{T}$ bases. The critical non-thermal stress protein sequences, the CTCT pyrimidines, were found to have the shortest UV stimulated fluorescence lifetimes. Their conjugate GAGA purines were found to have lifetimes that are approximately an order of magnitude longer.

Fluorescence lifetimes are measured in the picosecond time range, a relevant time scale for molecular rearrangements. Since the fluorescence lifetimes relate to the properties of the excited molecule and not to what caused the excitation, the measured UV excitation life- 
times in the picosecond range also applied to lifetimes of perturbations due to EMF. Therefore, the studies suggest that the conjugate segments on the DNA strands will retain energy for significantly different lengths of time. The shorter lasting perturbation is evidence of more rapid dissipation of energy through collisions and a greater likelihood of reaching a breaking point, while the rest of the chain is a being held in place by the conjugate strand.

A second paper [46] on the flexibility of the double DNA helix found that stretching fluctuations were much larger than expected and extended over two turns of the DNA helix (over 10 base pairs). The double helix is apparently much more flexible than would be expected from the multiple H-bond links between the two strands, and mechanical stresses are probably more easily transmitted along the strand. The flexibility of the DNA double helix would make the local unraveling of the two strands proceed more easily once it starts.

These recently described properties of the DNA double helix support the idea that CTCT sequences are likely to play a greater role in DNA strand separation leading to initiation of transcription. Similar responses can occur with other base sequences, but they would require greater energy and are less likely to occur at lower frequencies. At higher frequencies, electron movements are apt to be induced all along the DNA strands.

Another approach to mechanism has been proposed by Gordon [28]. According to this model DNA function is controlled by acoustic signals (phonons), which can be enhanced via stochastic summation, i.e use of background. 'noise' if required, to complete the signal at the necessary strength [28]. This idea proposes that protein iterative activities account for enzyme activation, channel completion, and other functions necessary for cell homeostasis, and have been an integral part of electromagnetic responsive elements nCTCTn. Thus the suggestion that paramagnetic/diamagnetic transduction (damping) is a first order mechanism in this dynamic centered around the Schrodinger equation [to create a binary phonon signal series is extant in the literature [28]. According to this theory, beta sub-units direct protein conformational adaptation in response to acoustic signal series to complete an EM driven control circuit capable of directing oxidation-reduction reactions (Ubbink et al. [67]. An alternate mechanism to variations on cyclotron resonance has been suggested by [16,17]. Gordon [28] and Panagopolus [50] suggest that EM fields may act as classical "forcers" in a resonance system with paramagnetic/diamagnetic oscillators that "damp" the EM field via transduction into a normal mode or elementary phonon compatible with the intrinsic design and length of the protein. This hypothesis suggests that displacement of electrons in the H-bonds that hold DNA together leading to DNA chain separation and initiating transcription are reflections of a phonon resonance/iterative process.

\subsection{Signaling Proteins}

How does all this work? In the case of Planaria the DNA in the totipotent stem cells created by the injury may be responding directly to the EM fields. DNA-mediated charge transport and the oxidative damage that results are extremely sensitive to variations in the sequence and conformational-adaptive response leading to stacking of the intervening bases [18]. Protein binding to DNA, for example, through one or more of the MAPKinase cascades, may modulates long-range charge transport negatively and positively depending upon the specific protein /DNA interactions in play [52,54].

Increased activation of the MAP-Kinase signaling pathways and the specific protein binding activities that normally occurs prior to upregulation of gene expression are increased by EMF $[26,34,38,72]$. Protein transcription factors in the p38 MAPK pathway are reported to be involved during both ELF and RF exposures [38]. Increased phosphorylation of specific transcription factors entering the nucleus, bind to specific regions of the recognition sites on the promoter and are essential for the initiation of transcription.

\subsection{Potential Biomedical Applications}

EM field exposures to induce hsp70 expression offer non-invasive methods to provide cytoprotection before, during and following surgical procedures or in areas of highly predictable trauma, e.g. combat, contact sports. In terms of potential biomedical applications of EM fields, it is intriguing to consider the data presented here together with our previous reports on cytoprotection and potential for gene therapy using a known specific DNA sequence that is responsive to EM fields, e.g. NGF, HSP70.

The stimulation of repair is a documented biological effect of EM fields [5]. However, some of the variability in results obtained may possibly be related to different responses to repair in different tissues, the exposure protocols employed and specific pulse parameters [29]. With modern lightweight designs prophylactic applications are technologically feasible, and could result in more rapid repair. Re-exposure at specific intervals would maintain the increased levels and provide increased protection.

Treatment with EM fields has been shown to protect and enhance injury repair in ischemia reperfusion injury [22] and enhanced regeneration of injured sciatic nerve, in both examples, after EM field exposure was discontinued [36]. Post-EM field exposure $(60 \mathrm{~Hz}, 80 \mathrm{mG}$ sine wave) of as little as 40 minutes, induces elevated hsp70 levels that were found to remain elevated for more than 
3 hours and remained capable of re-stimulation to the same or higher levels using a higher or lower field strength $[8,23,24]$. Gordon $[28,29]$ suggests that paramagnetic/diamagnetic dynamics in natively responsive elements generate quantum signal series that control conformational adaptation of proteins including DNA, enzymes and membrane proteins. He notes that identification of electromagnetic response elements in DNA was an important first insight and has proposed that DNA and especially promoter areas are highly responsive to these signal series. Dennis and Goodwin [19] examined PEMF technologies to define pulse bioefficacy and reported $\mathrm{dB} / \mathrm{dt}$ or "rise time" as the critical determinant within the PEMF technologies they examined. They went on to note, "rectangular pulses with rapid $\mathrm{dB} / \mathrm{dt}$ were up to four times more bio-effective in stimulating classes of genes associated with tissue restoration following trauma compared to DC, sine wave or millisecond designs". Our findings tend to corroborate that assessment, but also note the importance of specific frequencies within the parameters of our study.

\subsection{Summary and Conclusions}

In the experiments reported here, the flatworm Planaria Dugesia dorotocethala was used as a model system to assess whether frequencies that are multiples of eight induced regrowth and levels of stress response proteins (hsp70) quicker and with longer duration of effect when compared to a sinusoidal signal. There is good evidence, as previously suggested by Dennis and Goodwin [19] that the critical design determinant in pulsed electromagnetic field technologies in terms of bioefficacy is the $\mathrm{dB} / \mathrm{dt}$ or "rise time" in such designs and that rise time bioefficacy could be based upon electromagnetically responsive elements present in proteins, which would imbue the protein with highly sophisticated abilities to select harmonics necessary for homeostasis. The significance of effect vs. efficacy is largely avoided among designers and investigators of PEMF devices. The failure to address this question has resulted in highly variable results and criticism that PEMF technology lacks "scientific basis". This paper is the first in an attempt to discuss and evaluate specific frequencies in the ELFEMF range.

\section{ACKNOWLEDGEMENT}

Dedicated in loving memory to Abraham J Kremer and Glen A Gordon. We are grateful to Janet Wilson of Carolina Biologicals and Eve Vagg, Department of Photography and Illustration for their dedicated help.

\section{REFERENCES}

[1] K. Agata, (2003) Regeneration and gene regulation in planarians, Curr Opin Genet Dev, 13, 492-496.
[2] K. Agata, Y. Soejima, K. Kato, C. Kobayashi, Y. Umesono, and K. Watanabe, (1998) Structure of the planarian central nervous system (CNS) revealed by neuronal cell markers, Zool Sci, 15, 433-440.

[3] K. Agata and Y. Umesono, (2008) Brain regeneration from pluripotent stem cells in planarian, Phil. Trans. R. Soc. B, 363, 2071-2078.

[4] A. Albertini, P. Zucchini, G. Noera, R. Cadossi, C. P. Napoleone, and A. Pierangeli, (1999) Protective effect of low frequency low energy pulsing electromagnetic fields on acute experimental myocardial infarcts in rats.

[5] J. Baguna and R. Romero, (1981) Quantitative analysis of cell types during growth, regrowth and regeneration in the planarians digesia mediterranea and dugesia tigrina, Hydrobiologia, 84, 181-194.

[6] C. A. L. Bassett, (1995) Bioelectromagnetics in the service of medicine, Adv Chem, 250, 261-276.

[7] J. P. Blanchard and C. F. Blackman, (1994) Clarification and amplication of an ion parametric resonance model for magnetic field interaction with cells, Bioelectromagnetics, 14, 273-286.

[8] M. Blank and R. Goodman, (2007) A mechanism for stimulation of biosynthesis by electromagnetic fields: Charge transfer in DNA and base pair separation, J Cell Physiol doi10. 1002/jcp. 21198.

[9] M. Blank and R. Goodman, (2004) Initial interaction in electromagnetic field-induced biosynthesis, J Cell Physiol, 199, 359-363.

[10] M. Blank and R. Goodman, (1997) Do electromagnetic fields interact directly with DNA? Bioelectromagnetics, 18, 111-115.

[11] M. Blank and L. Soo, (2001) Electromagnetic acceleration of electron transfer reactions, J Cell Biochem, 81, 278-283.

[12] D. J. Canaday and R. C. Lee, (1991) Scientific basis for clinical applications of electric fields in soft tissue repair, In Electromagnetics in Medicine. (C. Brighton and S. Pollack, Eds.), San Francsico Press, San Francisco, CA, 275-292.

[13] S. Carmody, X. L. Wu, H. Lin, M. Blank, H. Skopicki, and R. Goodman, (2000) Cytoprotection by electromagnetic field-induced hsp70: A model for clinical application, J Cell Biochem, 79, 453-459.

[14] F. Cebria, T. Kudome, M. Nakazawa, K. Mineta, K. Ikeo, T. Gojobori, and K. Agata, (2002a) The expression of neural-specific genes reveals the structural and molecular complexity of the planarian central nervous system, Mech Dev, 116, 199-204.

[15] F. Cebria, M. Nakazawa, K. Mineta, K. Ikeo, T. Gojobori, and K. Agata, (2002b) Dissecting planarian central nervous system regeneration by the expression of neural-specific genes, Dev Growth Differ, 44, 135-146.

[16] K. Chang and W. R. Chang, (2003) Pulsed electromagnetic fields prevent osteoporosis in ovariectomized fe- 
male rat model: A prostaglandin E2 associated process, Bioelectromagnetics, 24, 189-198.

[17] K. C. Chou, (1988) Review: Low frequency collective motion in biomacromolecules and its biological functions, Biophysical Chemistry, 30, 3-48.

[18] K. C. Chou, (1984) Low frequency vibration of DNA molecules, Biochemical Journal, 221, 27-31.

[19] P. J. Dandliker, R. E. Holmlin, and J. K. Barton, (1997) Oxidative thymine repair in the DNA helix, Science, 275, 1465-1468.

[20] R. Dennis and T. Goodwin, (2003) Physiological and molecular genetics effects of time-varying electromagnetic fields on human neuronal cells, NASA Technical Paper TP-2003-212054.

[21] A. L. DiCarlo, J. M. Farrell, and T. A Litovitz, (1999) Myocardial protection conferred by electromagnetic fields, Circulation, 99, 813-816.

[22] C. Eichwald and J. Wallaczek, (1996) Activation dependent and biphasic electromagneetic field effects: Model based on cooperative enzyme kinetics in cellular signaling, Bioelectromagnetics, 17(6), 427-35.

[23] I. George, M. S. Geddis, Z. Lill, H. Lin, T. Gomez, M. Blank, M. C. Oz, and R. Goodman, (2008) Myocardial function improved by electromagnetic field induction of stress protein hsp70. J Cell Physiol DOI: 10. 1002/jcp 21461.

[24] R. Goodman and M. Blank, (2002) Insights into electromagnetic interaction mechanisms, J Cell Physiol, 192, 16-22.

[25] R. Goodman and M. Blank, (1998) Magnetic field stress induces expression of hsp70, Cell Stress/Chaperones 3, 79-88.

[26] R. Goodman and A. S. Henderson, (1988) Exposure of salivary gland cells to low-frequency electromagnetic fields alters polypeptide synthesis, Proc Natl Acad Sci U S A, 85, 3928-3932.

[27] R. Goodman, Y. A. Lin, M. Geddis, S. Hodge, S. Pantazatos, M. Blank, and R. Ambron, (2009) Electromagnetic fields activate the ERK cascade, increase hsp70 protein levels and promote regeneration in Planaria, International Journal of Radiation Biology, In Press.

[28] R. Goodman, D. Weisbrot, A. Uluc, and A. Henderson, (1992) Transcription in Drosophila melanogaster salivary gland cells is altered following exposure to low frequency electromagnetic fields: Analysis of chromosome 3R, Bioelectromagnetics, 13, 111-118.

[29] G. Gordon, (2008) Extrinsic electromagnetic fields, low frequency (phonon) vibrations and control of cell function: A non-linear resonance system, J Biomed Sci and Engineering, 1, 152-156.

[30] G. Gordon, (2007) Designed electromagnetic pulsed therapy: Clinical applications, J Cell Physiol, 212, 579582.

[31] L. Han, H. Lin, M. Head, M. Jin, M. Blank, and R.
Goodman, (1998) Application of magnetic field-induced heat shock protein 70 for presurgical cytoprotection, J Cell Biochem, 71, 577-583.

[32] H. Ito and C. A. Bassett, (1983) Effect of weak, pulsing electromagnetic fields on neural regeneration in the rat, Clin Orthop Relat Res, 181, 283-290.

[33] K. A. Jenrow, C. H. Smith, and A. R. Liboff, (1996) Weak extremely low frequency magnetic field-induced regeneration anomalies in the planarian dugesia tigrina, Bioelectromagnetics, 17, 467-474.

[34] K. A. Jenrow, C. H. Smith, and A. R. Liboff, (1995) Weak extremely low frequency magnetic fields and regeneration in the planarian dugesia tigrina, Bioelectromagnetics, 16, 106-112.

[35] M. Jin, M. Blank, and R. Goodman, (2000) ERK1/2 phosphorylation, induced by electromagnetic fields, diminishes during neoplastic transformation, J Cell Biochem, 78, 371-379.

[36] M. Jin, H. Lin, L. Han, M. Opler, S. Maurer, M. Blank, and R. Goodman, (1997) Biological and technical variables in $c$-myc expression in HL60 cells exposed to $60 \mathrm{~Hz}$ electromagnetic fields, Bioelectrochem Bioenerg, 44, $111-120$.

[37] M. Kanje, A. Rusovan, B. Sisken, and G. Lundborg, (1993) Pretreatment of rats with pulsed electromagnetic fields enhances regeneration of the sciatic nerve, Bioelectromagnetics, 14, 353-359.

[38] V. V. Lednev, (1991) Possible mechanism for influence of magnetic fields on biological systems, Bioelectromagnetics, 12, 71-75.

[39] D. Leszczynski, S. Joenvaara, J. Reivinen, and R. Kuokka, (2002) Non-thermal activation of the hsp27/ p38MAPK stress pathway by mobile phone radiation in human endothelial cells: molecular mechanism for cancer-and blood-brain barrier-related effects, Differentiation, 70, 120-129.

[40] A. Liboff, (2004) Toward and electromagnetic paradigm for biology and medicine, Journal of Alternative and Complementary Medicine, 1, 41-47.

[41] A. Liboff, (1985) Geomagnetic cyclotron resonance in membrane transport, J Biol Physics, 13, 99-102.

[42] H. Lin, M. Blank, M. Head, and R. Goodman, (1999) A magnetic field-responsive domain in the human HSP70 promoter, J Cell Biochem, 75, 170-176.

[43] H. Lin, M. Blank, K. R. Haseroth, and R. Goodman, (2001) Regulating genes with electromagnetic response elements, J Cell Biochem, 81, 143-148.

[44] H. Lin, L. Han, M. Blank, M. Head, and R. Goodman, (1998a) Magnetic field activation of protein-DNA binding, J Cell Biochem, 70, 297-303.

[45] H. Lin, M. Head, M. Blank, L. Han, M. Jin, R. Goodman, (1998b) Myc-mediated transactivation of HSP70 expression following exposure to magnetic fields, J Cell Biochem, 69, 181-188. 
[46] H. Lin, M. Opler, M. Head, M. Blank, and R. Goodman, (1997) Electromagnetic field exposure induces rapid, transitory heat shock factor activation in human cells, $\mathrm{J}$ Cell Biochem, 66, 482-488.

[47] R. S. M. Fenn, R. Das, and P. A. B. Harbury, (2009) Remeasuring the double helix, Science, 322, 449.

[48] R. I. Morimoto, (1998) Regulation of the heat shock transcriptional response: Cross talk between a family of heat shock factors, molecular chaperones and negative regulators, Genes \& Development.

[49] W. J. O'Brien, H. M. Murray, and M. G. Orgel, (1984) Effects of pulsing electromagnet fields on nerve regeneration: Correlation of electrophysiologic and histochemical parameters in adult guinea pigs, J Bioelectricity, 3, 33-40.

[50] M. G. Orgel, W. J. O'Brien, and H. M. Murray, (1984) Pulsing electromagnetic field therapy in nerve regeneration: an experimental study in the cat, Plast Reconstr Surg, 73, 173-183.

[51] D. J. Panagopoulos, A. Karabarbounis, and L. H. Margaritis, (2002) Mechanism of action of electromagnetic fields on cells, Biochem Biophys Res Com, 298, 95-102.

[52] A. R. Raji and R. E. Bowden, (1983) Effects of high-peak pulsed electromagnetic field on the degeneration and regeneration of the common peroneal nerve in rats, J Bone Joint Surg Br, 65, 478-492.

[53] S. Rajski and J. K. Barton, (2001) How different DNAbinding proteins affect long-range-oxidated damage to DNA, Biochemistry, 40, 5556-5564.

[54] S. Rao and A. S. Henderson, (1996) Regulation of c-fos is affected by electromagnetic fields, J Cell Biochem, 63, 358-365.

[55] M. Ratner, (1999) Electronic motion in DNA, Nature, 397, 480-481.

[56] E. Salo and J. Baguna, (1984) Regeneration and pattern formation in planarians, J Embryol, Exp Morphol, 83, 63-80.

[57] A. S. Alvarado, (2006) Planarian regeneration: Its ending is its beginning, Cell, 124, 241-245.

[58] A. A. Sanchez, P. A. Newmark, S. M. Robb, and R. Juste, (2002) The Schmidtea mediterranea database as a molecular resource for studying platyhelminths, stem cells and regeneration, Development, 129, 5659-5665.

[59] A. A. Sanchez and Newmark, (1999) Double-stranded RNA specifically disrupts gene expression during planarian regeneration, Proceedings of the National Academy of Sciences, USA; Developmental Biology, 96(9), 5049-5054.

[60] A. A. Sanchez and P. A. Newmark, (1998) The use of planarians to dissect the molecular basis of metazoan regeneration, Wound Rep Reg, 6, 413-420.
[61] N. Schwalb and F. Temps, (2008) Base sequence and higher-order structure induce the complex excited-state dynamics in DNA, Science, 322, 243-245.

[62] J. M. Shallom, A. L. Di Carlo, D. Ko, L. M. Penafiel, A. Nakai, and T. A. Litovitz, (2002) Microwave exposure induces Hsp70 and confers protection against hypoxia in chick embryos, J Cell Biochem, 86, 490-496.

[63] F. Shao, K. Augustyn, and J. K. Barton, (2005) Sequence dependence of charge transport through DNA domains, J Am Chem Soc, 127, 17445-17452.

[64] B. F. Sisken, M. Kanje, G. Lundborg, E. Herbst, and W. Kurtz, (1989) Stimulation of rat sciatic nerve regeneration with pulsed electromagnetic fields, Brain Res, 485, 309-316.

[65] B. F. Sisken, J. Walker, and M. Orgel, (1993) Prospects on clinical applications of electrical stimulation for nerve regeneration, J Cell Biochem, 51, 404-409.

[66] J. L. Walker, J. M. Evans, P. Meade, P. Resig, and B. F. Sisken, (1994a) Gait-stance duration as a measure of injury and recovery in the rat sciatic nerve model, $\mathrm{J}$ Neurosci Methods, 52, 47-52.

[67] J. L. Walker, J. M. Evans, P. Resig, S. Guarnieri, P. Meade, and B. F. Sisken, (1994b) Enhancement of functional recovery following a crush lesion to the rat sciatic nerve by exposure to pulsed electromagnetic fields, Exp Neurol, 125, 302-305.

[68] M. Ubbink, M. Ejdeback, B. G. Karlsson, and D. S. Bendall, (1998) The structure of the complex of plastocyanin and cytochrome $f$, determined by paramagnetic NMR and restrained rigid-body molecular dynamics, Structure, 3, 323-335.

[69] C. Wan, T. Fiebig, S. O. Kelley, C. R. Treadway, and J. K. Barton, (1999) Femtosecond dynamics of DNA mediated electron transfer, Proc Nat Acad Sci USA, 96, 6014-6019.

[70] C. Wan, T. Fiebig, O. Schiemann, J. K. Barton, and A. H. Zewail, (2000) Femtosecond direct observation of charge transfer between bases in DNA, Proc Natl Acad Sci USA, 97, 14052-14055.

[71] R. V. Weber, A. Navarro, J. K. Wu, H. L. Yu, and B. Strauch, (2004) Pulsed magnetic fields applied to a tranferred arterial loop support the rat groin composite flap, Plast Reconstr Surg, 114, 1185-1189.

[72] D. Weisbrot, O. Khorkova, H. Lin, A. Henderson, and R. Goodman, (1993) The effect of low frequency electric and magnetic fields on gene expression in Saccharomyces cerevisiae, Bioelectrochem Bioenerg, 31, 167-177.

[73] D. Weisbrot, H. Lin, L. Ye, M. Blank, and R. Goodman, (2003) Effects of mobile phone radiation on growth and development in Drosophila melanogaster, J Cell Biochem, 89, 48-55. 